\title{
Is the Vision of Radioligand Therapy for Prostate Cancer Becoming a Reality? An Overview of the Phase III VISION Trial and Its Importance for the Future of Theranostics
}

\author{
Kambiz Rahbar ${ }^{1}$, Lisa Bodei ${ }^{2}$, and Michael J. Morris ${ }^{3}$ \\ ${ }^{I}$ Department of Nuclear Medicine, University Hospital Muenster, Muenster, Germany; ${ }^{2}$ Department of Nuclear Medicine, Memorial \\ Sloan Kettering Cancer Center, New York, New York; and ${ }^{3}$ Department of Genitourinary Oncology Service and Medicine, Memorial \\ Sloan Kettering Cancer Center, New York, New York
}

Prostate-specific membrane antigen (PSMA) is of considerable interest as a target for diagnostics and therapy of prostate cancer patients. PSMA-targeted imaging has demonstrable value in guiding the management of the clinical evolution of prostatic cancer. The use of PSMA-targeted therapy using ${ }^{177}$ Lu-labeled PSMA-617 is similarly effective and is progressing toward approval. The phase III VISION trial represents the largest well-designed and executed study of a theranostic pair. This article provides an overview of the phase III trial and delineates the different study arms and their implications in the assessment of efficacy. The VISION (phase III) trial will provide data of critical value to the field of theranostics and especially the field of prostatic cancer management.

Key Words: prostate cancer; PSMA-targeted therapy; theranostics

J Nucl Med 2019; 60:1504-1506

DOI: 10.2967/jnumed.119.234054

\section{$\mathbf{P}$} most common cause of cancer-related death in men (1). Evolving diagnostic and therapeutic strategies for men with advanced-stage, metastatic, prostate cancer have revolutionized the field and are of major clinical and economic interest, with the potential to extend survival while maintaining quality of life. One such strategy involves the use of agents that combine diagnostic and therapeutic capabilities. These agents can identify the presence of a target on a patient's cancer and normal tissues, to enhance the likelihood of patient benefit and minimize needless exposure or normal-organ toxicity. Some term the development of these agents theranostics.

One molecular target of intense interest has been prostatespecific membrane antigen (PSMA), a transmembrane protein that was discovered over 25 years ago and has been the subject of intense investigation since (2-11). The ability to identify PSMAexpressing prostate cancer cells using a noninvasive imaging-based method, followed by administration of experimental therapy, has led to the development of ${ }^{68} \mathrm{Ga}$-PSMA-11, a diagnostic compound used

Received Jul. 16, 2019; revision accepted Aug. 19, 2019.

For correspondence or reprints contact: Kambiz Rahbar, Department of Nuclear Medicine, University Hospital Muenster, Albert-Schweitzer-Campus 1, D-48149 Münster, Germany.

E-mail: rahbar@uni-muenster.de

Published online Aug. 26, 2019.

COPYRIGHT (c) 2019 by the Society of Nuclear Medicine and Molecular Imaging. to select patients for experimental treatment with ${ }^{177} \mathrm{Lu}-\mathrm{PSMA}-617$ radioligand $(3,12,13)$.

Developed by the German Cancer Center in Heidelberg, PSMA617 labeled with ${ }^{177} \mathrm{Lu}$ has garnered attention as an experimental therapeutic compound. In case reports and published patient series, it has shown promising response rates and low toxicity in men with advanced prostate cancer, and in the German Multicenter Study, it showed a prostate-specific antigen decline of $50 \%$ and an overall response rate of $45 \%$ (14-17).

An Australian phase II study led by Michael Hofman that treated 30 metastasized castration-resistant prostate cancer (mCRPC) patients who had significant prior treatment exposures resulted in significant declines in prostate-specific antigen with minimal toxicity (18). On the basis of these promising data, ${ }^{68} \mathrm{Ga}-\mathrm{PSMA}-11$ and ${ }^{177} \mathrm{Lu}$-PSMA617 are now being evaluated in a trial called VISION, an international prospective open-label, randomized phase III study in men with PSMA-expressing mCRPC (ClinicalTrials.gov identifier NCT03511664).

\section{PHASE III VISION TRIAL}

The overall design of the trial, and its primary and secondary endpoints, are depicted in Figure 1. Patients are randomized on a 2:1 basis to receive either the best standard of care (SOC) along with ${ }^{177} \mathrm{Lu}-\mathrm{PSMA}-617$ or the best SOC alone.

Men with metastatic prostate cancer have access to an expanding armamentarium of life-prolonging agents that can be applied early in the clinical course of metastatic disease (e.g., docetaxel, enzalutamide, apalutamide, or abiraterone) or in the context of mCRPC. Indeed, men with mCRPC may have already received chemotherapy and yet still have an intact performance status, be candidates for further treatment, and be appropriate for clinical trials. In recognition of these numerous treatment options for mCRPC, including first- and second-generation androgen receptor axis-directed drugs, palliative maneuvers, and other SOCs, the VISION trial was designed knowing that there are many ways to care for the chemotherapyexposed mCRPC treatment population. Because of safety concerns, chemotherapy and radium were not considered to be SOCs for this trial population, as the dosing and side effects of tumor-directed radiotherapy in combination with these marrow-toxic agents have not yet been defined. Patients who require or are likely to benefit from second-line chemotherapy are not allowed in the trial.

\section{IMPORTANCE OF THE VISION TRIAL}

The trial design contains a subtle, but critical, feature. Namely, it does not compare the SOC to treatment with ${ }^{177} \mathrm{Lu}-\mathrm{PSMA}-617$. 


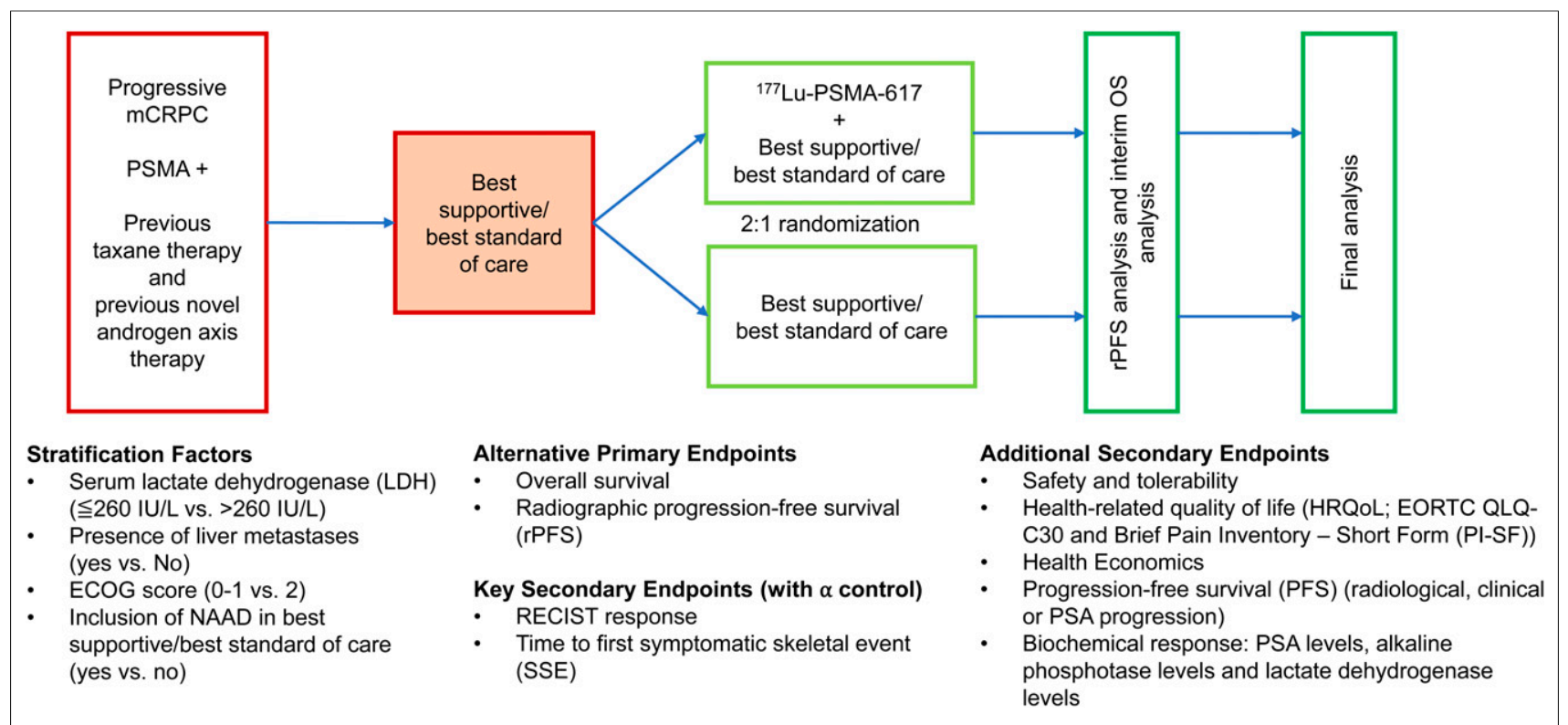

FIGURE 1. Overall trial design and primary and secondary endpoints. ECOG = Eastern Cooperative Oncology Group; EORTC QLQ-30 = European Organization for Research and Treatment of Cancer Quality-of-Life Core Questionnaire 30; NAAD = novel androgen axis drug; OS = overall survival.

The design compares the SOC $+{ }^{177} \mathrm{Lu}-\mathrm{PSMA}-617$ against the SOC. Thus, all therapeutic options, with the exception of cytotoxic, bone-targeted radiotherapy or other investigational treatments, can be used in either arm of the VISION trial. This leads to a critical issue. Patients who are in both arms of the trial must have expert care in the administration of systemic therapy for mCRPC and in the management of side effects of both the disease and the SOC systemic treatments, but this issue is especially relevant for patients in the control arm. For the trial to succeed, investigators must have a facile knowledge of SOC options available to the patients; the ability to manage end-stage prostate cancer patients using SOC options; and the ability to have a frank discussion with trial candidates regarding their expectations if assigned to the SOC-only arm. In addition, since many patients have seen data on the Internet suggesting that ${ }^{177}$ Lu-PSMA-617 can have an antitumor effect, investigators must impart a clear understanding that ${ }^{177} \mathrm{Lu}-\mathrm{PSMA}-617$ has not, to date, shown any survival advantage or other metric of clinical benefit over the SOC.

This last point is especially important. The VISION trial is the first international, randomized study testing the hypothesis that overall survival is increased after treatment with ${ }^{177} \mathrm{Lu}$-PSMA-617 in men with advanced-stage prostate cancer. The trial also contains an alternate primary endpoint of radiographic progressionfree survival whose integrity requires that patients assigned to the control arm continue receiving the SOC until such time as a clinical or radiographic progression-free survival event occurs. On the

\section{NOTEWORTHY}

- PSMA-targeted ligands have significantly changed the management of prostate cancer patients.

- The phase III VISION trial is needed to prove the benefit of this novel therapeutic according to current oncologic standards.

- Failure of the VISION trial would be a drawback for the entire field of theranostics. occurrence of such an event, these patients can then receive any and all options available to them, including other experimental therapy, but not ${ }^{177} \mathrm{Lu}-\mathrm{PSMA}-617$. Maintaining control arm patients in the study until the point of a radiographic progression-free survival event correlates with the speed at which the trial reaches the alternate primary endpoint; the more intact the integrity of the study remains, the faster will arrive the answer of whether ${ }^{177} \mathrm{Lu}-\mathrm{PSMA}-617$ fulfils its potential as a new therapeutic option.

If the VISION trial succeeds, it establishes a new line of therapy for prostate cancer that becomes the developmental paradigm for theranostics: the ability to use ${ }^{68}$ Ga-PSMA-617-based imaging to identify patients for treatment with ${ }^{177} \mathrm{Lu}-\mathrm{PSMA}-617$. If successful, it may become impossible to conduct any subsequent study testing whether the VISION target population benefits from ${ }^{177} \mathrm{Lu}-$ PSMA-617 in terms of overall survival. Alternatively, failure of the VISION trial-meaning that the largest well-designed and executed study of a theranostic pair has failed-would be a drawback for the entire field of theranostics. Independent of the activity of the drug, success or failure is in the hands of the clinical investigators and their ability to maintain the integrity of the VISION trial as it is designed.

\section{DISCLOSURE}

Kambiz Rahbar reports being a compensated consultant for $\mathrm{ABX}$ and has received consultant fees from Bayer and lectureship fees from Janssen Cielag, Amgen, AAA, and SIRTEX. Lisa Bodei is a consultant to AAA (compensation: hotel and dinner), Ipsen (noncompensated), and Curium (compensation: travel). Michael J. Morris is an uncompensated consultant for Astellas, Bayer, and Endocyte; is a compensated consultant for Advanced Accelerator Applications, Blue Earth Diagnostics, and Tokai; has institutional research contracts with Bayer, Sanofi, Endocyte, Progenics, Corcept, and Roche; and has received travel grants from Bayer, Endocyte, and Fujifilm. No other potential conflict of interest relevant to this article was reported. 


\section{REFERENCES}

1. Siegel RL, Miller KD, Jemal A. Cancer statistics, 2015. CA Cancer J Clin. 2015;65:5-29.

2. Afshar-Oromieh A, Babich JW, Kratochwil C, et al. The rise of PSMA ligands for diagnosis and therapy of prostate cancer. J Nucl Med. 2016;57(suppl 3): 79S-89S.

3. Afshar-Oromieh A, Holland-Letz T, Giesel FL, et al. Diagnostic performance of ${ }^{68}$ Ga-PSMA-11 (HBED-CC) PET/CT in patients with recurrent prostate cancer: evaluation in 1007 patients. Eur J Nucl Med Mol Imaging. 2017;44:1258-1268.

4. Rahbar K, Ahmadzadehfar H, Boegemann M. ${ }^{177}$ Lu-PSMA-617 radioligand therapy in mCRPC: ready for phase III trial? Eur J Nucl Med Mol Imaging. 2018;45:513-514.

5. Israeli RS, Powell CT, Corr JG, Fair WR, Heston WD. Expression of the prostate-specific membrane antigen. Cancer Res. 1994;54:1807-1811.

6. Afshar-Oromieh A, Hetzheim H, Kratochwil C, et al. The theranostic PSMA ligand PSMA-617 in the diagnosis of prostate cancer by PET/CT: biodistribution in humans, radiation dosimetry, and first evaluation of tumor lesions. J Nucl Med. 2015;56:1697-1705.

7. Kratochwil C, Giesel FL, Eder M, et al. ${ }^{177}$ Lu-lutetium-labelled PSMA ligandinduced remission in a patient with metastatic prostate cancer. Eur J Nucl Med Mol Imaging. 2015;42:987-988.

8. Kratochwil C, Giesel FL, Stefanova M, et al. PSMA-targeted radionuclide therapy of metastatic castration-resistant prostate cancer with ${ }^{177} \mathrm{Lu}$-labeled PSMA-617. J Nucl Med. 2016;57:1170-1176.

9. Fendler WP, Kratochwil C, Ahmadzadehfar H, et al. ${ }^{177}$ Lu-PSMA-617 therapy, dosimetry and follow-up in patients with metastatic castration-resistant prostate cancer [in German]. Nuklearmedizin.2016;55:123-128.
10. O'Keefe DS, Bacich DJ, Huang SS, Heston WDW. A perspective on the evolving story of PSMA biology, PSMA-based imaging, and endoradiotherapeutic strategies. J Nucl Med. 2018;59:1007-1013.

11. Baum RP, Kulkarni HR, Schuchardt C, et al. ${ }^{177} \mathrm{Lu}$-labeled prostate-specific membrane antigen radioligand therapy of metastatic castration-resistant prostate cancer: safety and efficacy. J Nucl Med. 2016;57:1006-1013.

12. Afshar-Oromieh A, Avtzi E, Giesel FL, et al. The diagnostic value of PET/CT imaging with the ${ }^{68} \mathrm{Ga}$-labelled PSMA ligand HBED-CC in the diagnosis of recurrent prostate cancer. Eur J Nucl Med Mol Imaging. 2015;42:197-209.

13. Fendler WP, Calais J, Eiber M, et al. Assessment of ${ }^{68} \mathrm{Ga}$-PSMA-11 PET accuracy in localizing recurrent prostate cancer: a prospective single-arm clinical trial. JAMA Oncol. 2019;5:856-863.

14. Ahmadzadehfar H, Rahbar K, Kurpig S, et al. Early side effects and first results of radioligand therapy with ${ }^{177} \mathrm{Lu}$-DKFZ-617 PSMA of castrate-resistant metastatic prostate cancer: a two-centre study. EJNMMI Res. 2015;5:114.

15. Rahbar K, Bode A, Weckesser M, et al. Radioligand therapy with ${ }^{177}$ Lu-PSMA617 as a novel therapeutic option in patients with metastatic castration resistant prostate cancer. Clin Nucl Med. 2016;41:522-528.

16. Rahbar K, Schmidt M, Heinzel A, et al. Response and tolerability of a single dose of ${ }^{177} \mathrm{Lu}$-PSMA-617 in patients with metastatic castration-resistant prostate cancer: a multicenter retrospective analysis. J Nucl Med. 2016;57:1334-1338.

17. Kessel K, Seifert R, Schäfers M, et al. Second line chemotherapy and visceral metastases are associated with poor survival in patients with $\mathrm{mCRPC}$ receiving ${ }^{177}$ Lu-PSMA-617. Theranostics. 2019;9:4841-4848.

18. Hofman MS, Violet J, Hicks RJ, et al. $\left[{ }^{177} \mathrm{Lu}\right]-P S M A-617$ radionuclide treatment in patients with metastatic castration-resistant prostate cancer (LuPSMA trial): a single-centre, single-arm, phase 2 study. Lancet Oncol. 2018;19:825-833. 